\title{
Interactive comment on "Calving Front Machine (CALFIN): Glacial Termini Dataset and Automated Deep Learning Extraction Method for Greenland, 1972-2019" by Daniel Cheng et al.
}

\section{Anonymous Referee \#1}

Received and published: 9 November 2020

\section{General Comments}

This manuscript introduces the novel developed Calving Front Machine "CALFIN" for the automated extraction of Greenlandic calving fronts. This is a major contribution to the field as it replaces time-consuming manual delineated fronts by automatically extracted dense glacier front time series. The CALFIN algorithm was validated extensively against test datasets and results from previous studies through a model intercomparison. The scientific community will definitely benefit from this development as an automatically derived calving front position data set of 66 Greenlandic glaciers will be released with this publication. 
Despite the impressive results and technical details of this manuscript, I have some concerns about the structure of this paper and the (sometimes) very short explanations. However, after re-structuring some parts of the manuscript and adding additional information as indicated below, this paper will present an important contribution to the field.

In my opinion, the abstract should be structured more clearly. For a better understanding, I would recommend to re-order the abstract by using the common schema: 1) Statement of the problem, 2) Research question, 3) Research design, 4) Central results, 5) Brief interpretation of the results, and 6) Outlook/ future use of the data set.

P2L4: The paper introduces a new method and provides an inter-comparison with other studies. For readers not familiar with the studies of Zhang et al, Mohajerani et al. and Baumhoer et al. it would be helpful to have a brief state-of-the-art paragraph reviewing existing calving front extraction methods. For example, P2L4 could be extended and give more insights into the studies used in the inter-comparison as well as the studies of Seale et al. 2011 and similar approaches.

P2L11: In my opinion this section is incomplete. Please mention all potential data sources in Table 1 (add Sentinel-2, Envisat, ERS, Radarsat) and justify why they are not suitable. Another option would be to just focus on Landsat data and remove the incomplete Table 1. Figure 1 is really great so I would try to put the focus on it and highlight the incredible amount of processed data and outline the advantages, data amount, and characteristics of Landsat.

P2L17: The methodology section could give a short overview of the entire workflow from pre-processing to the final extracted calving front by showing a flow chart. This would guide the reader through the methodology part and link the numerous subchapters of section 3. Besides, in my opinion, the training of the network explained in P12L2 should be part of the methodology and not subject to the discussion. 
P5 Figure 5c: How does the filtering of unconfident predictions work? Please describe this in the methodology section.

P6L1: Please outline the calving front re-processing in more detail. Does the reprocessing allow a higher spatial accuracy when re-processing a part of the image?

P6L16: How much smoothing of the extracted coastline is allowed and can this also decrease accuracy?

P8L1: How did you handle the issue that your network was trained for 3-channel RGB imagery but tested on 1-channel SAR data?

P8L18: What are the characteristics of those outlier glaciers and how many glaciers are defined as "outlier"?

P11L15: The information of this section could also be shifted to methodology. Then rename Chapter 5 to "CALFIN Dataset".

P10L4: But also mention the mean distance which is comparable here.

P10 Figure 11: How did you consider the fact that ice shelves are much bigger than glaciers? For example, in Figure 11 you show the Shackleton ice shelf. It is approx. $200 \mathrm{~km}$ wide and if you resample that to $224 \times 224$ pixels, one pixel for your validation would be $892 \mathrm{~m}$ compared to $40 \mathrm{~m}$ pixels in the original study by Baumhoer et al. 2019 . How did this influence the validation accuracy? For Zhang et al. you show that the use of higher resolution of TerraSAR-X data does not improve the mean distance accuracy (Figure 10).

P13 Figure 13: Can you explain why the PROMICE data set (2008/2009 and 2010/2011) shows twice a very different front position compared to the CALFIN data set?

P13L13: The model inter-comparison is only discussed for the study of Mohajerani et al. but validations were also done against the data sets of Zhang et al. and Baumhoer 
et al., hence those results should also be discussed.

Interactive comment on The Cryosphere Discuss., https://doi.org/10.5194/tc-2020-231, 2020.

Interactive

comment 